\title{
Duality theorem for inductive limit groups
}

\author{
Nobuhiko Tatsuuma
}

\begin{abstract}
In this paper, we show the so-called weak duality theorem of Tannaka type for an inductive limit-type topological group $G=\lim _{n \rightarrow \infty} G_{n}$ in the case where each $G_{n}$ is a locally compact group, and $G_{n}$ is embedded into $G_{n+1}$ homeomorphically as a closed subgroup. First, we explain what a weak duality theorem of Tannaka type is and explain the difference between the case of locally compact groups and the case of nonlocally compact groups. Then we introduce the concept "separating system of unitary representations (SSUR)," which assures the existence of sufficiently many unitary representations. The present $G$ has an SSUR. We prove that $G$ is complete. We give semiregular representations and their extensions for $G$. Using them, we deduce a fundamental formula about "birepresentation" on $G$. Combining these results, we can prove the weak duality theorem of Tannaka type for $G$.
\end{abstract}

\section{Introduction}

Let $G=\lim _{n \rightarrow \infty} G_{n}$ be an inductive limit-type topological group. We treat the case where each $G_{n}$ is a locally compact group and $G_{n}$ is embedded in $G_{n+1}$ homeomorphically as a closed subgroup. We say that such a group is a closed type (see Definition 3.1). About topologies and duality theorem of this group, some results were given in [1], [3], [5], and [6]. In the previous paper [4], we studied properties of such a group and obtained some family of unitary representations of it. Based on these results, we show in this paper that for these groups, the so-called weak duality theorem of Tannaka type is valid.

The weak Tannaka-type duality theorem for topological groups is stated as follows.

We take the set $\Omega \equiv\left\{D=\left(\mathcal{H}^{D}, T_{g}^{D}\right)\right\}$ of all unitary representations of a given topological group $K$, dimensions of which are bounded by $\max \left(\aleph_{0}, \# K\right)$. There exist operations between elements of $\Omega$ such as

(1) unitary equivalence: $D_{1} \sim_{W} D_{2}$ (W: intertwining operator),

(2) direct sum: $D_{1} \oplus D_{2}$,

(3) tensor product: $D_{1} \otimes D_{2}$,

(4) contragradient representation: $D \rightarrow \bar{D}$.

We consider an operator field $\boldsymbol{U} \equiv\left\{U^{D}\right\}_{D \in \Omega}$ on $\Omega$ satisfying the following:

Kyoto Journal of Mathematics, Vol. 54, No. 1 (2014), 51-73

DOI $10.1215 / 21562261-2400274$, (C) 2014 by Kyoto University

Received January 20, 2012. Revised December 3, 2012. Accepted December 4, 2012.

2010 Mathematics Subject Classification: Primary 22A25; Secondary 22D35. 
(B-0) for each $D \in \Omega, U^{D}$ is a unitary operator on the representation Hilbert space $\mathcal{H}^{D}$

(B-1) $D_{1} \sim_{W} D_{2} \Longrightarrow W U^{D_{1}} W^{-1}=U^{D_{2}}$;

(B-2) $U^{D_{1}} \oplus U^{D_{2}}=U^{D_{1} \oplus D_{2}}$;

(B-3) $U^{D_{1}} \otimes U^{D_{2}}=U^{D_{1} \otimes D_{2}}$;

(B-4) $\overline{\left(U^{D}\right)}=U^{\bar{D}}$.

Here $\bar{D}$ is the contragradient representation of $D$, and $\overline{\left(U^{D}\right)}$ is the operator on $\mathcal{H}^{\bar{D}}$ "contragradient" to $U^{D}$ (see Section 1 ).

We shall call such an operator field $\boldsymbol{U}$ a birepresentation of $K$.

On the space of all birepresentations, we give a topology which is the product of weak topologies on spaces of unitary operators on Hilbert spaces $\mathcal{H}^{D}$.

As it is shown in Section 1, Lemma 1.1 that for any $g$ in $K$ the operator field $\boldsymbol{T}_{g} \equiv\left\{T_{g}^{D}\right\}_{D \in \Omega}$ satisfies the conditions (B.1)-(B.4); therefore $\boldsymbol{T}_{g}$ gives a birepresentation.

The weak Tannaka-type duality theorem asserts the converse.

\section{ASSERTION}

For any birepresentation $\boldsymbol{U} \equiv\left\{U^{D}\right\}_{D \in \Omega}$,

$$
\exists_{1} g \in K \quad \text { such that } U^{D}=T_{g}^{D} \quad(\forall D \in \Omega) .
$$

Moreover, the topology given above on the space of birepresentations coincides with the original topology of $K$ under this correspondence.

As shown in our previous paper [2], for any locally compact group, a theorem of such type is valid. But there exist several differences for the definition of birepresentation.

In duality theory for locally compact groups, birepresentations are operator fields $\boldsymbol{U} \equiv\left\{U^{D}\right\}_{D}$ with values not only of unitary operators but also of nonzero bounded operators on $\mathcal{H}^{D}$ at $D \in \Omega$. However, for the case of nonlocally compact groups, the unitary assumption cannot be abridged.

The reason for this phenomenon is explained as follows.

In the case of a locally compact group, norms of any component operators $U^{D}$ of birepresentation $\boldsymbol{U}$ are bounded by 1 (see [2, (2.16)]).

So, for the case where we take this bounded operator condition as the definition of birepresentation instead of unitarity, we shall call this type of duality for topological groups the $B$-type. Consider the space of operator fields

$$
\boldsymbol{B} \equiv\left\{\mathcal{B}=\left\{B^{D}\right\}_{D \in \Omega} \mid B^{D} \text { bounded operator on } \mathcal{H}^{D} \text { such that }\left\|B^{D}\right\| \leq 1\right\}
$$

with product topology of weak ones. The ball $\boldsymbol{B}^{D} \equiv\left\{B^{D} ;\left\|B^{D}\right\| \leq 1\right\}$ is weakly compact for each $D$. So the space $\boldsymbol{B}$, which is the direct product of these balls, is weakly compact too. The set $K_{U}$ of all birepresentations is a subgroup of $\boldsymbol{B}$.

Suppose, for a topological group $K$, that the $B$-type duality theorem is valid. Then we ask what can we conclude on the topology of $K$. 
It is easy to see that operations (1)-(4) are continuous with respect to the weak topology. The set $\boldsymbol{B}_{0} \subset \boldsymbol{B}$ satisfying conditions (B-1)-(B-4) is closed in $\boldsymbol{B}$ and hence is weakly compact. So $K$ is imbedded into $\boldsymbol{B}_{0}$ as $K \ni g \rightarrow \boldsymbol{T}_{g} \equiv$ $\left\{T_{g}^{D}\right\}_{D \in \Omega} \in \boldsymbol{B}_{0}$ with the isomorphic image $K_{U}$.

The only difference between $K_{U}$ and $\boldsymbol{B}_{0}$ is the nonzero condition. So we see $K_{U}=\boldsymbol{B}_{0} \backslash\{0\}$. Now we conclude that $K$ is locally compact as a set taken off one point from a compact set.

\section{PROPOSITION 0.1}

A topological group $K$ for which the B-type duality is valid must be a locally compact group.

\section{NOTATION}

For a representation $D=\left\{\mathcal{H}^{D}, T_{g}^{D}\right\}$ of $G$, we take its cyclic subrepresentation on the closed subspace $\left(\mathcal{H}^{D}\right)$ of $\mathcal{H}^{D}$ spanned by $\left\{T_{g}^{D} v^{D}\right\}_{g \in G}\left(v^{D} \in \mathcal{H}^{D},\left\|v^{D}\right\|=1\right)$. We express it as $(D)=\left\{\left(\mathcal{H}^{D}\right), T_{g}^{D}, v^{D}\right\}$.

Hereafter, for two cyclic representations $D_{j}=\left\{\mathcal{H}^{j}, T_{g}^{j}, v^{j}\right\}, j=1,2$, we denote cyclic representations contained in $D_{1} \oplus D_{2}$ and $D_{1} \otimes D_{2}$, respectively, as

$$
\begin{aligned}
\left(D_{1} \oplus D_{2}\right) & \equiv\left\{\left(\mathcal{H}^{1} \oplus \mathcal{H}^{2}\right), T_{g}^{1} \oplus T_{g}^{2}, v^{1} \oplus v^{2}\right\}, \\
\left(D_{1} \otimes D_{2}\right) & \equiv\left\{\left(\mathcal{H}^{1} \otimes \mathcal{H}^{2}\right), T_{g}^{1} \otimes T_{g}^{2}, v^{1} \otimes v^{2}\right\} .
\end{aligned}
$$

This paper is organized as follows. In Section 1, we introduce the concept separating system of unitary representations (SSUR), which assures existence of sufficiently many unitary representations. $G$ has an SSUR (see Section 1, Example 2).

To prove the completeness of $G$ in Section 4, we prepare lemmata about $G$ in Sections 2 and 3.

We give semiregular representations and their extensions of $G$ in Section 5 . Using results in Section 5, we deduce important properties about "birepresentations" on $G$ in Section 6 .

Combining results in Sections 4 and 6, we can prove the weak duality theorem of Tannaka type for $G$ in Section 7 .

In Section 8, we introduce a new notion, isobirepresentation, which gives a wider category than of birepresentation. We can prove an analogous duality theorem for these isobirepresentations.

\section{Separating system of unitary representations}

Consider a Hausdorff (i.e., $T_{2^{-}}$) topological group $K$.

Let $D \equiv\left\{\mathcal{H}^{D}, T_{g}^{D}, v^{D}\right\}$ be a cyclic unitary representation of $K$. Here $\mathcal{H}^{D}$ is the representation space, $T_{g}^{D}$ is the operator of representation, and $v^{D}$ is the normalized cyclic vector $\left\|v^{D}\right\|=1$. Then the function $\eta^{D}(g) \equiv\left\langle T_{g}^{D} v^{D}, v^{D}\right\rangle$ is a normalized continuous positive definite function on $K$. 
For later arguments, we treat $\bar{D} \equiv\left\{\overline{\mathcal{H}}, \bar{T}_{g}, \bar{v}\right\}$, the so-called contragradient representation of $D \equiv\left\{\mathcal{H}, T_{g}, v\right\}$, where $\overline{\mathcal{H}}$ is the linear dual space of $\mathcal{H}$, and there exists an antilinear map from $\mathcal{H}$ to $\overline{\mathcal{H}}$ defined by

$$
\mathcal{H} \ni u \rightarrow \bar{u} \in \overline{\mathcal{H}}: \quad \bar{u}(w) \equiv(w, \bar{u})=\langle w, u\rangle .
$$

In $\overline{\mathcal{H}}$, we introduce an inner product as $\langle\bar{w}, \bar{u}\rangle=\overline{\langle w, u\rangle}$. As is easily shown, $\overline{(\bar{u})}=u$. For a bounded operator $A$ on $\mathcal{H}$, we define $\bar{A} \bar{u} \equiv \overline{(A u)}$; then $\bar{A}$ gives a bounded linear operator on $\overline{\mathcal{H}}$. By definition, $\overline{T_{g}} \bar{u}=\overline{\left(T_{g} u\right)}$, and if $A$ and $B$ are bounded operators on $\mathcal{H}$, then $\overline{A B} \bar{u}=\bar{A}(\bar{B} \bar{u})=\bar{A} \overline{(B u)}=\overline{(A(B u))}=\overline{(A B u)}$.

Hence $g \rightarrow \bar{T}_{g} \equiv \overline{\left(T_{g}\right)}$ gives a unitary representation of $K$ on $\overline{\mathcal{H}}$. This is by definition the contragradient representation of $D$. Let $\bar{v} \in \overline{\mathcal{H}}$ be the vector corresponding to $v \in \mathcal{H}$ as above; then

$$
\forall g \in K, \quad\left\langle\bar{T}_{g} \bar{v}, \bar{v}\right\rangle=\overline{\left\langle T_{g} v, v\right\rangle} .
$$

It is easy to see that $\overline{(\bar{D})}$ is equivalent to the original $D$.

\section{LEMMA 1.1}

We have $\forall g \in K, \forall D \in \Omega, \overline{\left(T_{g}^{D}\right)}=T_{g}^{\bar{D}}$.

\section{Proof}

It is just the definition of $\bar{D}$.

On the other hand, the representation $D^{0} \equiv D \oplus \bar{D}$ is self-adjoint; that is, $\overline{\left(D^{0}\right)} \sim_{W} D^{0}$, with an intertwining operator $W$ exchanging the first space with the second space, which maps a vector $u \oplus \bar{v}$ to $v \oplus \bar{u}$.

\section{LEMMA 1.2}

Consider a representation $D^{0} \equiv D \oplus \bar{D}$ for a given representation $D \equiv\left\{\mathcal{H}^{D}, T_{g}^{D}\right\}$. Let $A$ be a bounded operator on $\mathcal{H}^{D}$.

Then, for $\forall u, v \in \mathcal{H}^{D},\langle(A \oplus \bar{A})(u \oplus \bar{u}), v \oplus \bar{v}\rangle(\leq 1)$ is real valued.

\section{Proof}

We have $\langle(A \oplus \bar{A})(u \oplus \bar{u}), v \oplus v\rangle=\langle A u, v\rangle+\langle\bar{A} \bar{u}, \bar{v}\rangle=\langle A u, v\rangle+\langle\overline{(A u)}, \bar{v}\rangle=$

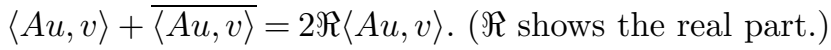

Now for any given cyclic unitary representation $D \equiv\left\{\mathcal{H}, T_{g}, v\right\}(\|v\|=1)$, and the trivial representation $I=\left\{\boldsymbol{C}, I_{g}, v_{0}\right\}$, we consider unitary representation

$$
D_{p} \equiv I \oplus D \oplus \bar{D}
$$

and its cyclic part $\left(D_{p}\right)$, whose representation space is spanned by the vector

$$
v_{p} \equiv\left(2^{-1 / 2}\right) v_{0} \oplus(1 / 2)(v \oplus \bar{v}) .
$$

COROLLARY 1.2.1

For the above cyclic representation $\left(D_{p}\right)$, the matrix element

$$
1 \geq\left\langle T_{g}^{D_{p}} v_{p}, v_{p}\right\rangle \geq 0
$$


Proof

At first, $\left\|v_{p}\right\|^{2}=2^{-1}\left\|v_{0}\right\|^{2}+2 \times 2^{-2}\|v\|^{2}=2^{-1}+2 \times 2^{-2}=1$.

Therefore $\left\langle T_{g}^{D_{p}} v_{p}, v_{p}\right\rangle \leq 1$ and $\left|\left\langle T_{g}^{D} v, v\right\rangle\right| \leq 1$,

$$
\begin{aligned}
1 & \geq\left\langle T_{g}^{D_{p}} v_{p}, v_{p}\right\rangle=\left(2^{-1}\right)\left\langle I_{g} v_{0}, v_{0}\right\rangle+\left(4^{-1}\right)\left\{\left\langle T_{g}^{D} v, v\right\rangle+\left\langle T_{g}^{\bar{D}} \bar{v}, \bar{v}\right\rangle\right\} \\
& =2^{-1}+\left(2^{-1}\right)\left(\Re\left\langle T_{g}^{D} v, v\right\rangle\right) \geq 0 .
\end{aligned}
$$

Write $\eta(g) \equiv\left\langle T_{g}^{D} v, v\right\rangle$. Take $0<\varepsilon<1$, and put

$$
F(D, \varepsilon) \equiv\{g \in K|| 1-\eta(g) \mid<\varepsilon\} .
$$

\section{COROLLARY 1.2.2}

Let $D$, and $\left(D_{p}\right)$ be as in Corollary 1.2.1. Then

$$
1>\forall \varepsilon \geq 0, \exists \delta>0, \quad F\left(D_{p}, \delta\right) \subset F(D, \varepsilon) .
$$

Proof

$$
|1-\eta(g)|<\varepsilon<1 \text { shows that }|1-\Re \eta(g)|<1 ; \quad \text { therefore } \Re \eta(g)>0 .
$$

Since $\eta_{p}(g)=\left\langle T_{g}^{D_{p}} v_{p}, v_{p}\right\rangle=2^{-1}\left(1+\left(\Re\left\langle T_{g}^{D} v, v\right\rangle\right)\right.$,

$$
1-\eta_{p}(g)=1-\Re \eta_{p}(g)=1-2^{-1}-\left(2^{-1}\right) \Re \eta(g)=2^{-1}(1-\Re \eta(g)) .
$$

On the other hand, $1 \geq|\Re \eta(g)|^{2}+|\Im \eta(g)|^{2}$. ( $\Im$ is the imaginary part.)

So $|\Im \eta(g)|^{2} \leq 1-(\Re \eta(g))^{2}=(1-\Re \eta(g)) \times(1+\Re \eta(g)) \leq 2(1-\Re \eta(g))$. Thus

$$
\begin{aligned}
|1-\eta(g)|^{2} & =|1-\Re \eta(g)|^{2}+|\Im \eta(g)|^{2} \leq(1-\Re \eta(g))^{2}+2(1-\Re \eta(g)) \\
& =(1-\Re \eta(g))(3-\Re \eta(g)) \leq 3(1-\Re \eta(g))=6\left(1-\eta_{p}(g)\right) .
\end{aligned}
$$

This shows that if $6 \delta<\varepsilon^{2}$, then $\forall g \in F\left(D_{p}, \delta\right)$ leads us to $g \in F(D, \varepsilon)$.

\section{DEFINITION 1.1}

We say that a set $\Omega_{0} \equiv\left\{D_{\alpha}\right\}_{\alpha \in A}$ of cyclic unitary representations of $K$ gives a separating system of unitary representations (SSUR) if, for any neighborhood $V$ of the unit $e$ in $K$, there exists a positive definite function $\eta^{D}(g) \equiv$ $\left\langle T_{g}^{D} v^{D}, v^{D}\right\rangle\left(D \in \Omega_{0}\right)$ and $\varepsilon>0$ such that

$$
\left\{g \in K|| 1-\eta^{D}(g) \mid<\varepsilon\right\} \subset V .
$$

\section{EXAMPLE 1}

Let $K$ be a locally compact group. For any neighborhood $V$ of $e$, we can take a continuous function $f$ such that $\left[f^{*} * f\right] \subset V$, where $\left[f^{*} * f\right]$ denotes the carrier of the convolution function of $f^{*}$ and $f$, where $f^{*}(g)=\Delta(g) \overline{f\left(g^{-1}\right)}(\Delta(g)$ shows the modular function on $G$ ).

As is well known, $f^{*} * f$ gives a positive definite function. This shows that the family of cyclic subrepresentations of the regular representation gives an SSUR of $K$. 


\section{EXAMPLE 2}

In [4, Proposition 5.5], we have shown that, for a closed-type inductive limit group $G=\lim _{n \rightarrow \infty} G_{n}$ and a neighborhood $V$ of $e$ in $G$, we can construct a positive definite function $F$ on $G$ such that the carrier $[F] \subset V$. This shows that the family of representations given in the paper [4] gives an SSUR for $G$.

\section{LEMMA 1.3}

If a topological group $K$ has an SSUR, then we can select a new SSUR $\Omega_{1} \equiv\{D\}$, for which any $D=\left\{\mathcal{H}^{D}, T_{g}^{D}, v^{D}\right\} \in \Omega_{1}$ has a nonnegative valued positive definite function $\eta^{D}(g) \equiv\left\langle T_{g}^{D} v^{D}, v^{D}\right\rangle$.

\section{Proof}

Take, instead of the initially given SSUR, $\Omega \equiv\{D\}$, the new system $\Omega_{1} \equiv\left\{\left(D_{p}\right)\right\}$ as above; Corollaries 1.2.1 and 1.2.2 show that $\Omega_{1}$ is also an SSUR.

For a given Hilbert space $\mathcal{H}$, we denote by $B(\mathcal{H})$ the space of all bounded operators, by $J(\mathcal{H})$ the space of all isometric operators, and by $U(\mathcal{H})$ the space of all unitary operators on $\mathcal{H}$. Put the weak topologies on $J(\mathcal{H})$ 's.

For $U \in J(\mathcal{H})$ and $v \in \mathcal{H}$,

$$
\begin{aligned}
\|U v-v\|^{2} & =\|U v\|^{2}+\|v\|^{2}-2 \Re\langle U v, v\rangle \\
& =2(\langle v, v\rangle-\Re(\langle U v, v\rangle))=2 \Re(\langle v-U v, v\rangle) .
\end{aligned}
$$

That is, the weak topology coincides with the strong topology.

Moreover, $U(\mathcal{H})$ becomes a topological group with the multiplication of operators and this topology. As a group topology, this topology gives a uniform structure on $U(\mathcal{H})$.

Now, for a topological group $K$, we consider any unitary representation $D \equiv$ $\left\{\mathcal{H}^{D}, T_{g}^{D}\right\}$ and the map $K \ni g \rightarrow T_{g}^{D} \in U\left(\mathcal{H}^{D}\right)$. Of course this map is continuous for each $D$.

Construct $\boldsymbol{U}(\Omega) \equiv \prod_{D \in \Omega} U\left(\mathcal{H}^{D}\right)$ with natural product topology. Then $\boldsymbol{U}(\Omega)$ is a topological group too, by the componentwise multiplication. The map

$$
K \ni g \longmapsto\left(T_{g}^{D}\right)_{D \in \Omega} \in \prod_{D \in \Omega} U\left(\mathcal{H}^{D}\right)=\boldsymbol{U}(\Omega)
$$

is an injective homomorphism as topological groups.

Now let $K$ be a $T_{2}$-topological group with an SSUR.

\section{LEMMA 1.4}

For this group $K$ the map (1.6) is an injective isomorphism, so by this map, $K$ is embedded as a topological group in $\boldsymbol{U}(\Omega)$.

\section{Proof}

According to the above discussions, this map is continuous.

Conversely the $T_{2}$-property and existence of an SSUR $\Omega_{0}$ show that this map must be injective, and for any neighborhood $V$ of $e \in K$, we can select elements 
$D \in \Omega_{0}$ and $\varepsilon>0$ such that

$$
\left\{g \in K \mid\left\|T_{g}^{D} v-v\right\|<\varepsilon\right\} \subset V .
$$

This shows the continuity of the inverse map of (1.6).

Hereafter we denote by $K_{U}$ the image of $K$ under (1.6) in $\boldsymbol{U}(\Omega)$.

\section{Cauchy filter base}

\section{DEFINITION 2.1}

Take a filter base $\mathcal{F} \equiv\left\{F_{\alpha}\right\}_{\alpha \in \Gamma}$ on a $T_{2}$-topological group $K$, where $\Gamma$ is a partially ordered set. We say that $\mathcal{F}$ is $l$-Cauchy (resp., $r$-Cauchy) if for any neighborhood $V$ of $e \in K$, there exists $\alpha \in \Gamma$ such that

$$
\left.\forall \beta, \gamma \succ \alpha(\beta, \gamma \in \Gamma), \quad F_{\beta}^{-1} F_{\gamma} \subset V \quad \text { (resp., } F_{\beta} F_{\gamma}^{-1} \subset V\right) .
$$

And we say that $\mathcal{F}$ is $b$-Cauchy (both Cauchy) when $\mathcal{F}$ is $l$-Cauchy and at the same time $\mathcal{F}^{-1} \equiv\left\{F_{\alpha}^{-1}\right\}_{\alpha \in \Gamma}$ is $l$-Cauchy.

If any $l$-Cauchy (resp., $r$-Cauchy, $b$-Cauchy) filter bases have limit points in $K$, we say that $K$ is $l$-complete (resp., $r$-complete, $b$-complete).

As is well known that an l-complete group is also $r$-complete.

For simplicity, hereafter we use the word "Cauchy" (resp., complete) for " $l$ Cauchy" (resp., l-complete).

A $b$-Cauchy filter base is also $l$-Cauchy, so an $l$-complete group is $b$-complete too.

If a filter base $\mathcal{F}$ converges to a point $g_{0}$ in $K$, then $\mathcal{F}^{-1}$ converges to $g_{0}^{-1}$.

\section{LEMMA 2.1}

For an arbitrary given Cauchy filter base $\mathcal{F} \equiv\left\{F_{\alpha}\right\}_{\alpha \in \Gamma}$ on a $T_{2}$-topological group $K$, the set $\overline{\mathcal{F}} \equiv\left\{\overline{F_{\alpha}}\right\}_{\alpha \in \Gamma}$ gives a base of a Cauchy filter on $K$ too, where $\overline{F_{\alpha}}$ denotes the closure of $F_{\alpha}$.

If one of $\mathcal{F}$ and $\overline{\mathcal{F}}$ converges, then the other one converges to the same limit point. (We say that this property is cofinal.)

Proof

Since $\overline{F_{\alpha}} \cap \overline{F_{\beta}} \supset \overline{F_{\alpha} \cap F_{\beta}}, \overline{\mathcal{F}} \equiv\left\{\overline{F_{\alpha}}\right\}_{\alpha \in \Gamma}$ gives a base of a filter on $K$.

We show that it is Cauchy.

For any given neighborhood $W$ of $e$, take a symmetric neighborhood $V$ (i.e., $\left.V=V^{-1}\right)$ of $e$ as $V^{3} \subset W$.

Because $\mathcal{F}$ is Cauchy, $\exists \alpha \in \Gamma$ such that $\forall \beta, \gamma \succ \alpha, F_{\beta}^{-1} F_{\gamma} \subset V$.

But $\overline{F_{\alpha}} \subset F_{\alpha} V, \overline{F_{\beta}} \subset F_{\beta} V$. Therefore ${\overline{F_{\alpha}}}^{-1} \overline{F_{\beta}} \subset V F_{\alpha}^{-1} F_{\beta} V \subset V^{3} \subset W$.

We have $\forall \alpha, \overline{F_{\alpha}} \supset F_{\alpha}$, so if $\overline{\mathcal{F}}$ converges, then $\mathcal{F}$ converges to the same limit.

Conversely if $\mathcal{F}$ converges, then $\mathcal{V} \mathcal{F} \equiv\left\{V F_{\alpha}\right\}_{\alpha \in \Gamma, V \in \mathcal{V}}(\mathcal{V}$ is the set of all neighborhoods of $e$ ) converges to the same limit.

Since $\mathcal{V} \mathcal{F} \supset \overline{\mathcal{F}}$, this concludes the proof. 


\section{DEFINITION 2.2}

We call a filter base $\mathcal{F} \equiv\left\{F_{\alpha}\right\}$ such that all $F_{\alpha}$ are closed, as C-filter base.

\section{Partially compact set in an inductive limit group}

DEFINITION 3.1

We say that an inductive limit group $G=\lim _{n \rightarrow \infty} G_{n}$ of closed type if each $G_{n}$ is a locally compact group, and $G_{n}$ is imbedded in $G_{n+1}$ homeomorphically as a closed subgroup.

\section{REMARK}

Here we can assume without any loss of generality that each $G_{n}$ is not locally isomorphic to $G_{n+1}$.

If there is an $n$ such that $\forall m \geq n, G_{m}$ is locally isomorphic to $G_{n}$, then $G$ is itself locally compact group which is locally isomorphic to $G_{n}$. So we have no need to discuss this case.

If not, we can take a subsequence $\left\{G_{n(m)}(n(m)<n(m+1))\right\}$ such that $G_{n(m)}$ is not locally isomorphic to $G_{n(m+1)}$, and $G$ is isomorphic to $\lim _{n \rightarrow \infty} G_{n(m)}$, which satisfies the above condition.

In this section, we consider a closed-type inductive limit group $G=\lim _{n \rightarrow \infty} G_{n}$.

We quote some results from our previous paper [4].

\section{DEFINITION 3.2}

Take for each $n$ a neighborhood $W_{n}$ of $e$ in $G_{n}$. Then

$$
W \equiv \bigcup_{1 \leq k<\infty} W_{1} \cdot W_{2} \cdots W_{k}
$$

is a neighborhood of $e$ in $G$. We call a neighborhood of this type a BS (bamboo shoot) neighborhood.

In [4, Proposition 2.3], the next proposition was shown.

PROPOSITION 3.1

Let $G$ be a closed-type inductive limit group. Then the family of BS neighborhoods gives a fundamental system of neighborhoods of $e$ in $G$.

\section{DEFINITION 3.3}

A subset $E(\subset G)$ is called a partially compact set, or a PC set, if for any $n, E \cap G_{n}$ is a compact set (may be vacant).

The next is obvious.

LEMMA 3.1

For any $P C$ set $E$ and $\forall g \in G$, the sets $E g, g E$ are also $P C$ sets. 
LEMMA 3.2

Let $E$ be a $P C$ set in $G$ such that $\exists n, E \cap G_{n}=\emptyset$. Then there exists a neighborhood $W$ of e in $G$ such that $E \cap G_{n} W=\emptyset$.

Proof

Since $G_{n}$ is closed in $G_{n+1}$, for the compact set $E_{n+1} \equiv E \cap G_{n+1}$, we can take a compact neighborhood $W_{n+1}$ of $e$ in $G_{n+1}$ such that $E_{n+1} \cap G_{n} W_{n+1}=\emptyset$.

Obviously $G_{n} W_{n+1} \subset G_{n+1}, G_{n}$ is closed, and $W_{n+1}$ is compact, so its product $G_{n} W_{n+1}$ is closed.

For a closed set $G_{n} W_{n+1}$ in $G_{n+2}$ and a compact set $E_{n+2} \equiv E \cap G_{n+2}$,

$$
\begin{aligned}
E_{n+2} \cap G_{n} W_{n+1} & =E \cap G_{n+2} \cap G_{n} W_{n+1}=E \cap G_{n+1} \cap G_{n} W_{n+1} \\
& =E_{n+1} \cap G_{n} W_{n+1}=\emptyset .
\end{aligned}
$$

So we can take a compact neighborhood $W_{n+2}$ of $e$ in $G_{n+2}$ such that

$$
E_{n+2} \cap G_{n} W_{n+1} W_{n+2}=\emptyset .
$$

Similarly for the closed set $G_{n} W_{n+1} W_{n+2}$ in $G_{n+3}$ and a compact set $E_{n+3} \equiv$ $E \cap G_{n+3}$, define a compact neighborhood $W_{n+3}$ of $e$ in $G_{n+3}$ such that

$$
E_{n+3} \cap G_{n} W_{n+1} W_{n+2} W_{n+3}=\emptyset .
$$

Repeating these steps, by induction on $k$, we can obtain a compact neighborhood $W_{n+k}$ such that

$$
E_{n+k} \cap G_{n} W_{n+1} W_{n+2} \cdots W_{n+k}=\emptyset .
$$

We have $\forall k>m, E_{n+k} \supset E_{n+m}, E_{n+m} \cap G_{n} W_{n+1} W_{n+2} \cdots W_{n+k}=\emptyset$, that is,

$$
E_{n+m} \cap G_{n}\left(\bigcup_{k \geq 1} W_{n+1} W_{n+2} \cdots W_{n+k}\right)=\emptyset .
$$

The set $W \equiv \bigcup_{k \geq 1} W_{n+1} W_{n+2} \cdots W_{n+k}$ is a BS neighborhood of $e$ in $G$, and

$$
\forall m, \quad E_{n+m} \cap G_{n} W=\emptyset .
$$

But $E=\bigcup_{k \geq 1} E_{n+k}$, and so $E \cap G_{n} W=\emptyset$.

LEMMA 3.3

If there exists a family $\left\{F_{m}\right\}_{m \geq 1}$ of $P C$ sets in $G$ satisfying

$$
\text { (1) } \forall m, \quad F_{m} \supset F_{m+1}, \quad(2) \quad \forall m, \quad F_{m} \cap G_{m}=\emptyset,
$$

then we can take a neighborhood $V$ of $e \in G$ such that

$$
\forall m, \quad F_{m+1} \cap G_{m} V=\emptyset .
$$

\section{Proof}

Take $F_{m+1}$ as $E$ in Lemma 3.2; then there exists a neighborhood $V_{m+1}$ of $e \in G$ such that $F_{m+1} \cap G_{m} V_{m+1}=\emptyset$. Here $G_{m} V_{m+1}$ is a neighborhood of $e \in G$. 
Put $V \equiv \bigcap_{m \geq 1} G_{m} V_{m+1}$. We show that $V$ is also a neighborhood of $e \in G$.

For this it is enough to show that $V$ contains an open neighborhood of $e \in G$. Since $V_{m}$ is a neighborhood in $G$, there exists an open neighborhood $O_{m} \subset V_{m}$ of $e \in G$. Put

$$
V=\bigcap_{m \geq 1} G_{m} V_{m+1} \supset O \equiv \bigcap_{m \geq 1} G_{m} O_{m+1} \ni e .
$$

To prove that $O$ is open in $G$, it is enough to see that $\forall k, O \cap G_{k}$ is open in $G_{k}$. But $\forall m \geq k, G_{k} \subset G_{m} O_{m+1}$. So

$$
\begin{aligned}
O \cap G_{k} & =\left(\bigcap_{m<k} G_{m} O_{m+1}\right) \cap\left(\bigcap_{m \geq k} G_{m} O_{m+1}\right) \cap G_{k} \\
& =\left(\bigcap_{m<k} G_{m} O_{m+1}\right) \cap\left(\left(\bigcap_{m \geq k} G_{m} O_{m+1}\right) \cap G_{k}\right) \\
& =\bigcap_{m<k} G_{m} O_{m+1} \cap G_{k},
\end{aligned}
$$

which is open in $G_{k}$. And

$$
\forall m, \quad F_{m+1} \cap G_{m} V=F_{m+1} \cap G_{m}\left(\bigcap_{k \geq 1} G_{k} V_{k+1}\right) \subset F_{m+1} \cap G_{m} V_{m+1}=\emptyset .
$$

\section{LEMMA 3.4}

For any neighborhood $V$ of $e \in G$, there exists a $P C$ neighborhood of $e \in G$ contained in $V$.

\section{Proof}

Without any loss of generality, we can assume that $V$ is a BS neighborhood; that is, we can consider it as $V=\bigcup_{1 \leq k<\infty} V_{1} \cdot V_{2} \cdots V_{k}$.

We will take $W_{n}$ in (3.1) inductively as $\forall n, W_{n} \subset V_{n}$.

At first, take an open relatively compact neighborhood of $e$ in $G_{1}$ as $W_{1} \subset V_{1}$.

Next, we select $W_{2}$ a relatively compact neighborhood of $e$ in $G_{2}$ as $W_{2} \subset V_{2}$ and $\left(W_{2}\right)^{2} \cap G_{1} \subset W_{1}$.

Now, if we can determine $W_{j-1}$, then we select $W_{j}$ as a relatively compact neighborhood of $e$ in $G_{j}$ satisfying $W_{j} \subset V_{j}$ and $\left(W_{j}\right)^{2} \cap G_{j-1} \subset W_{j-1}$.

In this situation $W_{1} \cdot W_{2} \cdots W_{j-1} \cdot\left(W_{j}\right)^{2}$ is a relatively compact neighborhood of $e$ in $G_{j}$. Now put $W \equiv \bigcup_{k \geq 1} W_{1} \cdot W_{2} \cdots W_{k}$ and $E(k, j) \equiv W_{1} \cdot W_{2} \cdots W_{k} \cap G_{j}$.

If $k \leq j$, then $G_{j} \supset W_{1} \cdot W_{2} \cdots W_{k} \supset E(k, j)$.

When $k>j$, then

$$
\begin{aligned}
E(k, j) & \equiv W_{1} \cdot W_{2} \cdots W_{k} \cap G_{j} \subset W_{1} \cdot W_{2} \cdots W_{k-1}\left(W_{k}\right)^{2} \cap G_{j} \\
& =W_{1} \cdot W_{2} \cdots W_{k-1}\left(W_{k}\right)^{2} \cap G_{k-1} \cap G_{j} \\
& =\left(W_{1} \cdot W_{2} \cdots W_{k-1}\left(W_{k}\right)^{2} \cap G_{k-1}\right) \cap G_{j} \\
& \subset\left(\left(W_{1} \cdot W_{2} \cdots W_{k-1}\right)\left(\left(W_{k}\right)^{2} \cap G_{k-1}\right)\right) \cap G_{j}
\end{aligned}
$$




$$
\begin{aligned}
& \subset W_{1} \cdot W_{2} \cdots W_{k-2}\left(W_{k-1}\right)^{2} \cap G_{k-2} \cap G_{j} \\
& \cdots \\
& \subset W_{1} \cdot W_{2} \cdots W_{j-1}\left(W_{j}\right)^{2} \cap G_{j}=W_{1} \cdot W_{2} \cdots W_{j-1}\left(W_{j}\right)^{2} .
\end{aligned}
$$

In both cases, $\forall k, E(k, j) \subset W_{1} \cdot W_{2} \cdots W_{j-1}\left(W_{j}\right)^{2}$, that is,

$$
W \cap G_{j} \equiv \bigcup_{k \geq 1} W_{1} \cdot W_{2} \cdots W_{k} \cap G_{j} \subset W_{1} \cdot W_{2} \cdots W_{j-1}\left(W_{j}\right)^{2}
$$

is a relatively compact set in $G$.

Since $W$ is a neighborhood of $e$ in the topological group $G$, there exists a neighborhood $V_{0}$ of $e$ such that $\left(V_{0}\right)^{2} \subset W$ and $\overline{V_{0}} \subset\left(V_{0}\right)^{2} \subset W$.

Thus $\overline{V_{0}}$ is closed, and $\forall n, \overline{V_{0}} \cap G_{n}\left(\subset W \cap G_{n}\right)$ is compact.

$\overline{V_{0}}$ is a $\mathrm{PC}$ neighborhood of $e \in G$ contained in $V$.

\section{COROLLARY 3.4.1}

If $\mathcal{F}=\left\{F_{\alpha}\right\}_{\alpha \in A}$ is a Cauchy $C$-filter base, then there exists an $\alpha$ such that for all $\beta \succ \alpha, F_{\beta}$ are $P C$ sets.

\section{Proof}

Take a PC neighborhood $W$ of $e \in G$. Since $\mathcal{F}=\left\{F_{\alpha}\right\}_{\alpha \in A}$ is Cauchy, $\exists \alpha, \forall \beta>$ $\alpha, F_{\alpha}^{-1} F_{\beta} \subset W$. So, for some $g \in F_{\alpha}, F_{\beta} \subset g W$. This shows that $\forall \beta>\alpha, F_{\beta}$ is a $\mathrm{PC}$ set.

\section{LEMMA 3.5}

A $\sigma$-compact set in a Hilbert space is contained in a closed separable subspace.

Proof

Any compact set $C$ in a metric vector space has a countable dense subset $\Xi$.

For a given $\sigma$-compact set $B \equiv \bigcup_{n \geq 1} C_{n}$ with $C_{n}$ compact, take $\Xi_{n}$ a countable dense set in $C_{n}$. Then $\bigcup_{n \geq 1} \Xi_{n}$ spans a closed separable subspace containing $B$.

\section{COROLLARY 3.5.1}

Let $E$ be a partially compact set in $G$.

For any unitary representation $D=\left\{\mathcal{H}^{D}, T_{g}^{D}\right\}$ of $G$ and $v \in \mathcal{H}^{D}$, the set $T_{E}^{D} v \equiv\left\{T_{g}^{D} v \mid g \in E\right\}$ is contained in a closed separable subspace.

\section{Proof}

The set $E$ is $\sigma$-compact, so its continuous image $T_{E}^{D} v$ is the same.

4. Completeness of $G=\lim _{n \rightarrow \infty} G_{n}$

\section{THEOREM 1}

A closed-type inductive limit group $G=\lim _{n \rightarrow \infty} G_{n}$ is complete. 
Proof

We show that any Cauchy filter base $\mathcal{F} \equiv\left\{F_{\alpha}\right\}_{\alpha \in A}$ on $G$ converges to a point in $G$.

There exist two cases.

Case (1) We have $\exists n$ such that $\forall \alpha, F_{\alpha} \cap G_{n} \neq \emptyset$;

Case (2) We have $\forall n, \exists \alpha$ such that $F_{\alpha} \cap G_{n}=\emptyset$.

In case (1), the set $\mathcal{F}_{n} \equiv\left\{F_{\alpha, n} \equiv F_{\alpha} \cap G_{n}\right\}_{\alpha \in A}$ gives a Cauchy filter base in the locally compact group $G_{n}$. In fact,

$$
\forall F_{\alpha, n}, F_{\beta, n} \in \mathcal{F}_{n}, \quad F_{\alpha, n} \cap F_{\beta, n}=\left(F_{\alpha} \cap G_{n}\right) \cap\left(F_{\beta} \cap G_{n}\right)=\left(F_{\alpha} \cap F_{\beta}\right) \cap G_{n}
$$

contains an element of $\mathcal{F}_{n}$. So $\mathcal{F}_{n}$ gives a filter base. The "Cauchy property" for $\mathcal{F}$ assures the same property for $\mathcal{F}_{n}$.

Since a locally compact group is complete, $\mathcal{F}_{n}$ converges to a point in $G_{n}$. This point is also the limit of $\mathcal{F}$. So $\mathcal{F}$ converges to a point of $G_{n}$.

Now we shall show that case (2) does not exist.

Assume that $\mathcal{F}$ is a $C$-filter base as in case (2). By Corollary 3.4.1, all elements of $\mathcal{F}$ can be taken as PC sets. Since $G=\bigcup G_{n}$, for $\forall F_{\alpha}, \exists n$ such that

$$
F_{\alpha} \cap G_{n} \neq \emptyset \text {. }
$$

Now take $F_{1} \in \mathcal{F}$ as $F_{1} \cap G_{1}=\emptyset$ and $n(1)$ as $F_{1} \cap G_{n(1)} \neq \emptyset$.

Next take $F_{2} \in \mathcal{F}$ as $F_{2} \subset F_{1}, F_{2} \cap G_{n(1)}=\emptyset$, and $n(2)$ as $F_{2} \cap G_{n(2)} \neq \emptyset$. take

Repeating these steps inductively, after determining $F_{k-1}$ and $n(k-1)$, we

$$
F_{k} \in \mathcal{F} \quad \text { as } F_{k} \subset F_{k-1}, F_{k} \cap G_{n(k-1)}=\emptyset, \quad \text { and } \quad n(k) \quad \text { as } F_{k} \cap G_{n(k)} \neq \emptyset .
$$

Thus we obtain a sequence $\left\{F_{m}, n(m)\right\}_{m \geq 1}$ of pairs as

$$
\text { (4.2) } \forall m, \quad F_{m+1} \subset F_{m}, \quad F_{m+1} \cap G_{n(m)}=\emptyset, \quad F_{m+1} \cap G_{n(m+1)} \neq \emptyset .
$$

For any inductive limit group $G=\lim _{n \rightarrow \infty} G_{n}$, we can omit components in the middle of the sequence. So we rewrite $G_{n(m)}$ to $G_{m}$, and apply Lemma 3.3. Then we obtain a neighborhood $V$ of $e \in G$ satisfying $\forall m, F_{m+1} \cap G_{m} V=\emptyset$. In other words,

$$
G_{m} F_{m+1} \cap V=\emptyset \quad \text { and } \quad F_{m+1} \cap G_{m+1} \neq \emptyset .
$$

Now, we quote here the result of [5, Proposition 5.5, Theorem 5.10].

\section{PROPOSITION}

Let $G=\lim _{n \rightarrow \infty} G_{n}$ be a closed-type inductive limit group. Then for any neighborhood $V$ of e in $G$, there exists a continuous positive definite function $\eta$ such that its support is contained in $V$ :

$$
[\eta] \subset V \text {. }
$$

Recall that the GNS-construction method gives a cyclic unitary representation $D \equiv\left\{\mathcal{H}, T_{g}, v\right\}$ such that $\eta(g)=\left\langle T_{g} v, v\right\rangle$, and (4.4) means that, if $g_{0}$ is not con- 
tained in $V$, then $\left\langle T_{g_{0}} v, v\right\rangle=0$; that is the same as

$$
T_{g_{0}} v \perp v .
$$

Combining with (4.3), we get

$$
\forall h_{m} \in G_{m}, \forall g_{m+1} \in F_{m+1}, \quad T_{h_{m}^{-1} g_{m+1}} v \perp v,
$$

that is,

$$
\forall h_{m} \in G_{m}, \forall g_{m+1} \in F_{m+1}, \quad T_{g_{m+1}} v \perp T_{h_{m}} v .
$$

On the other hand, the family of sets of vectors $\left\{T_{F} v\right\}_{F \in \mathcal{F}}$ must be a Cauchy filter base in the representation Hilbert space $\mathcal{H}$. Let $u$ be the limit of $\left\{T_{F} v\right\}_{F \in \mathcal{F}}$. Now put

$$
D_{m} \equiv\left\{g \in G \mid\left\|T_{g} v-u\right\|<1 / m\right\} .
$$

Since $u$ is the limit of monotone filter base $\left\{T_{F_{m}} v\right\}_{m \geq 1}$, we have $\forall m, n$, $D_{m} \cap F_{n} \neq \emptyset$.

Put $E(m) \equiv D_{m} \cap F_{m}$. Then these sets are nonempty and monotone decreasing with respect to $m$. By $(4.2), G_{m} \cap E(m+1)=\emptyset$.

At first, we take the minimal $n(1)$ such that $G_{n(1)} \cap E(1) \neq \emptyset$ and take $g_{1} \in G_{n(1)} \cap E(1)$.

Next take the minimal $n(2)$ such that $G_{n(2)} \cap E(n(1)+1) \neq \emptyset$ and $g_{2} \in$ $G_{n(2)} \cap E(n(1)+1)$.

Repeating this process, take the minimal $n(k)$ such that $G_{n(k)} \cap E(n(k-$ $1)+1) \neq \emptyset$, we obtain a sequence of pairs $\left\{\left(n(k), g_{k}\right)\right\}_{k \geq 1}$, where $g_{k} \in G_{n(k)} \cap$ $E(n(k-1)+1)$. Since $G_{n}$ are monotone increasing,

$$
\forall m<k, \quad g_{m} \in G_{n(k)-1}, \quad \text { but } \quad g_{k} \in E(n(k-1)+1) \subset F(n(k-1)+1) .
$$

The equation (4.7) claims $\forall m<k, T_{g_{m}} v \perp T_{g_{k}} v$; that is, all the elements of the sequence $\left\{T_{g_{k}} v\right\}_{k \geq 1}$ are mutually orthogonal. But we say that the family of sets of vectors $\left\{T_{F} v\right\}_{F \in \mathcal{F}}$ must be a Cauchy filter base. This is a contradiction.

\section{Semiregular representation}

In the previous paper [5, Sections 5.1-5.3, Theorem 5.10], for any given PC neighborhood $E$ of $e \in G$, we construct explicitly a cyclic unitary representation of $G$ corresponding to a positive definite function $\eta(g) \equiv\left\langle R_{g} f^{\sim}, f^{\sim}\right\rangle$ such that the support of $\eta$ satisfies $[\eta] \subset E$. We write this unitary representation as $\mathfrak{R} \equiv$ $\left\{\mathfrak{H}, R_{g}, f^{\sim}\right\}$, and review its construction.

First we select a sequence $\left\{\left(f_{n}^{\sim}, \mu_{n}\right)\right\}_{n \geq 1}$ of pairs of positive-valued continuous function $f_{n}^{\sim}$ and right Haar measure $\mu_{n}$ on $G_{n}$, inductively.

The vector $f^{\sim}$ is given as a continuous positive-valued function on $G$ whose restriction on each $G_{n}$ is in $L^{2}\left(\mu_{n}\right)$, and $f^{\sim}$ is the uniform limit and at the same time the limit in $L^{2}\left(\mu_{n}\right)$ of $f_{n}^{\sim}, n \geq 1$. 
We show

$$
\begin{aligned}
\left\|f^{\sim}\right\| & \equiv \lim _{n \rightarrow \infty}\left\|f_{n}^{\sim}\right\|_{L^{2}\left(\mu_{n}\right)} \\
& =\lim _{n \rightarrow \infty}\left(\int_{G_{n}}\left|f_{n}^{\sim}(g)\right|^{2} d \mu_{n}(g)\right)^{1 / 2}=1, \\
\left\|R_{g} f^{\sim}\right\| & \equiv \lim _{n \rightarrow \infty}\left\|R_{g} f_{n}^{\sim}\right\|_{L^{2}\left(\mu_{n}\right)}=\left\|f^{\sim}\right\| \quad(\forall g \in G), \\
\left(\left\|R_{g_{1}} f_{n}^{\sim}\right\|_{L^{2}\left(\mu_{n}\right)}\right)^{2} & =\int_{G_{n}}\left|R_{g_{1}} f_{n}^{\sim}(g)\right|^{2} d \mu_{n}(g) \quad\left(\forall g_{1} \in G_{n}\right) .
\end{aligned}
$$

Here $R_{g}$ denotes the right translation by $g$ on a function.

Consider the space $\boldsymbol{H}$ linearly spanned by $\left\{R_{g} f^{\sim}\right\}_{g \in G}$, that is, the space of functions $\left\{\sum_{j} c_{j} R_{g_{j}} f^{\sim}(g)\right\}$ on $G$. The norm $\|*\|$ gives a pre-Hilbert space structure on $\boldsymbol{H}$, and its completion $\mathfrak{H}$ is a Hilbert space, and $\mathfrak{R} \equiv\left\{\mathfrak{H}, R_{g}, f^{\sim}\right\}$ is a unitary representation of $G$ such that $[\eta] \subset E$ for $\eta(g) \equiv\left\langle R_{g} f^{\sim}, f^{\sim}\right\rangle$. Thus the construction is reviewed.

\section{DEFINITION 5.1}

We call the representation $\mathfrak{R} \equiv\left\{\mathfrak{H}, R_{g}, f^{\sim}\right\}$, a semiregular representation.

However, in the following, we will be forced to treat other unitary representations.

Take a cyclic unitary representation $D \equiv\left\{\mathcal{H}^{D}, T_{g}^{D}, v^{D}\right\}$ of $G$, and take a cyclic part of the tensor product

$$
D^{\sim} \equiv(D \otimes \mathfrak{R})=\left\{\left(\mathcal{H}^{D} \otimes \mathfrak{H}\right), T_{g}^{D} \otimes R_{g}, \boldsymbol{f}^{\sim} \equiv v^{D} \otimes f^{\sim}\right\},
$$

where $(D \otimes \mathfrak{R})$ means the subrepresentation of $D \otimes \mathfrak{R}$ on the subspace $\left(\mathcal{H}^{D} \otimes \mathfrak{H}\right)$ of $\mathcal{H}^{D} \otimes \mathfrak{H}$ spanned by $\boldsymbol{f}^{\sim}$. As is easily shown, an element of $\mathcal{H}^{D} \otimes \boldsymbol{H}$ is considered as a vector-valued function $\boldsymbol{f}(g) \equiv \sum_{j} c_{j} R_{g_{j}} f^{\sim}(g) v_{j}\left(v_{j} \in \mathcal{H}^{D}\right)$ on $G$, and for $\boldsymbol{f}, \boldsymbol{k} \in \mathcal{H}^{D} \otimes \boldsymbol{H}$

$$
\begin{aligned}
\|\boldsymbol{f}\|^{2} & =\lim _{n \rightarrow \infty} \int_{G_{n}}\|\boldsymbol{f}(g)\|_{\mathcal{H}^{D}}^{2} d \mu_{n}(g), \\
\langle\boldsymbol{f}, \boldsymbol{k}\rangle & =\lim _{n \rightarrow \infty} \int_{G_{n}}\langle\boldsymbol{f}(g), \boldsymbol{k}(g)\rangle_{\mathcal{H}^{D}} d \mu_{n}(g) .
\end{aligned}
$$

Then the representation $D^{\sim}$ belongs to the positive definite function

$$
\begin{aligned}
\left\langle T_{g}^{D^{\sim}} \boldsymbol{f}^{\sim}, \boldsymbol{f}^{\sim}\right\rangle & =\left\langle\left(T_{g}^{D} \otimes R_{g}\right)\left(v^{D} \otimes f^{\sim}\right),\left(v^{D} \otimes f^{\sim}\right)\right\rangle \\
& =\left\langle T_{g}^{D} v^{D}, v^{D}\right\rangle \cdot\left\langle R_{g} f^{\sim}, f^{\sim}\right\rangle .
\end{aligned}
$$

As a product of two continuous functions, $\left\langle T_{g}^{D^{\sim}} \boldsymbol{f}^{\sim}, \boldsymbol{f}^{\sim}\right\rangle$ is continuous. So the "direct product" representation $D^{\sim}$ is also continuous.

Elements of $\mathcal{H}^{D} \otimes \mathfrak{H}$ are considered to be vector-valued functions on $G$.

We consider an operator $\left(T_{g}^{0} \boldsymbol{f}\right)(*) \equiv T_{g}^{D} \boldsymbol{f}(* g)$ on this space; then

$$
D^{(0)} \equiv\left\{\mathcal{H}^{D} \otimes \mathfrak{H}, T_{g}^{0}\right\}
$$


gives a unitary representation of $G$. As the restriction to the subspace $\left(\mathcal{H}^{D} \otimes \mathfrak{H}\right)$ of $\mathcal{H}^{D} \otimes \mathfrak{H}, D^{\sim}$ is a subrepresentation of $D^{(0)}$.

The vector $\boldsymbol{f}^{\sim}=v^{D} \otimes f^{\sim}$ is represented as $f^{\sim}(g) v^{D}$, and $T_{g}^{0}\left(v^{D} \otimes f^{\sim}\right)=$ $f^{\sim}(* g)\left(T_{g}^{D} v^{D}\right)$.

Consider an operator

$$
W: \boldsymbol{f}(g) \rightarrow T_{g}^{D} \boldsymbol{f}(g)
$$

on $\mathcal{H}^{D} \otimes \mathfrak{H}$, that is, for $\boldsymbol{f}^{\sim} \equiv v^{D} \otimes f^{\sim}, W \boldsymbol{f}^{\sim}(g)=T_{g}^{D} v^{D} \otimes f^{\sim}(g)$, and

$$
\begin{aligned}
\left\|W \boldsymbol{f}^{\sim}\right\|_{\mathcal{H}^{D} \otimes \mathfrak{H}} & =\left\|T_{*}^{D} \boldsymbol{f}^{\sim}(*)\right\|_{\mathcal{H}^{D} \otimes \mathfrak{H}} \\
& =\lim _{n \rightarrow \infty}\left(\int_{G_{n}}\left\|f_{n}^{\sim}(g) T_{g}^{D} v^{D}\right\|^{2} d \mu(g)\right)^{1 / 2} \\
& =\lim _{n \rightarrow \infty}\left(\int_{G_{n}}\left|f_{n}^{\sim}(g)\right|^{2}\left\|v^{D}\right\|^{2} d \mu(g)\right)^{1 / 2} \\
& =\lim _{n \rightarrow \infty}\left(\int_{G_{n}}\left\|f_{n}^{\sim}(g) v^{D}\right\|^{2} d \mu(g)\right)^{1 / 2}=\left\|\boldsymbol{f}^{\sim}\right\|_{\mathcal{H}^{D} \otimes \mathfrak{H}} .
\end{aligned}
$$

Moreover, $T_{g^{-1}}^{D}=\left(T_{g}^{D}\right)^{-1}$, so $W$ gives a unitary operator. Therefore $D^{1} \equiv\left\{\mathcal{H}^{D} \otimes\right.$ $\left.\mathfrak{H}, W T_{g}^{D^{0}} W^{-1}\right\}$ is a unitary representation of $G$ and is equivalent to $D^{0}$. The relation

$$
\begin{aligned}
W T_{g_{0}}^{D^{0}} W^{-1}\left(T_{*}^{D}\left(v^{D}\right) \otimes f^{\sim}(*)\right) & =W\left(T_{g_{0}}^{D^{0}} v^{D} f^{\sim}\left(* g_{0}\right)\right) \\
& =W\left(\left(T_{* g_{0}}^{D} v^{D}\right) f\left(* g_{0}\right)\right)=\boldsymbol{f}\left(* g_{0}\right)
\end{aligned}
$$

shows that the operator $T_{g}^{D^{1}} \equiv W T_{g}^{D^{0}} W^{-1}$ is the right translation operator by $g$ in this representation $D^{1} \equiv\left\{\mathcal{H}^{D} \otimes \mathfrak{H}, T_{g}^{1}\right\}$.

We take $E$ as a PC set in $G$. By Corollary 3.5.1, $T_{E}^{D} v^{D}$ is contained in a closed separable subspace $\mathcal{H}_{0}^{D}$ in $\mathcal{H}^{D}$. We fix a $\operatorname{CONS}\left\{v_{j}\right\}$ in $\mathcal{H}_{0}^{D}$ such as $v_{1}=v^{D}$.

Now expand with respect to this $\operatorname{CONS}\left\{v_{j}\right\}, \boldsymbol{f}$ in $\mathcal{H}^{D}$ as

$$
\boldsymbol{f}(*)=\sum_{j \geq 1}\left\langle\boldsymbol{f}(*), v_{j}\right\rangle v_{j} ;
$$

then,

$$
\left(T_{g}^{1} \boldsymbol{f}\right)(*)=\sum_{j \geq 1}\left\langle\boldsymbol{f}(* g), v_{j}\right\rangle v_{j} \quad(g \in E) .
$$

This means that for each $j$ the space $\boldsymbol{H}_{j} \equiv\left\{\left\langle\boldsymbol{f}(*), v_{j}\right\rangle v_{j}\right\}_{\boldsymbol{f} \in\left(\mathcal{H}^{D} \otimes \mathfrak{H}\right)}$ is an invariant subspace in $\mathcal{H}^{D}$ for any $f$ such that $[f] \subset E$.

We return to our $D^{\sim}$. According to the above arguments, $W\left(f^{\sim}(g) v^{D}\right)=$ $f^{\sim}(g) T_{g}^{D} v^{D}$ for any $g \in G$, and $f^{\sim}(g)=0$ if $g \notin E$, so the components in (5.10) are

$$
\left\langle\boldsymbol{f}(*), v_{j}\right\rangle=f^{\sim}(*)\left\langle T_{*}^{D} v^{D}, v_{j}\right\rangle .
$$

Especially in the case $j=1$, we have $\left\langle\boldsymbol{f}(*), v^{D}\right\rangle=f^{\sim}(*)\left\langle T_{*}^{D} v^{D}, v^{D}\right\rangle$. 
The subrepresentation corresponding to this component is realized on a function space $\mathfrak{H}_{D}$ on $G$, spanned by $\left\{R_{g}\left(f^{\sim}(*)\left\langle T_{*}^{D} v^{D}, v^{D}\right\rangle\right)\right\}_{g \in G}$, and the operators of representation are the right translation $R_{g}$ on this function space.

\section{DEFINITION 5.2}

We call the representation

$$
D^{\sim}(D) \equiv\left\{\left(\mathfrak{H}_{D}\right), R_{g}, f^{\sim}(*)\left\langle T_{*}^{D} v^{D}, v^{D}\right\rangle\right\}
$$

of $G$ a generalized semiregular representation.

\section{Birepresentation of $G$}

Now we remark on some elementary properties of birepresentations.

From the condition (B-4), for any birepresentation $\boldsymbol{U} \equiv\left\{U^{D}\right\}$,

$$
U^{\bar{D}}=\overline{\left(U^{D}\right)} \text {. }
$$

\section{LEMMA 6.1}

For $D^{0} \equiv D \oplus \bar{D},\left\langle U^{D^{0}}(u \oplus \bar{u}), v \oplus \bar{v}\right\rangle$ is real valued.

Proof

We have

$$
\begin{aligned}
\left\langle U^{D^{0}}(u \oplus \bar{u}), v \oplus \bar{v}\right\rangle & =\left\langle U^{D} u, v\right\rangle+\left\langle U^{\bar{D}} \bar{u}, \bar{v}\right\rangle \\
& =\left\langle U^{D} u, v\right\rangle+\left\langle\overline{\left(U^{D} u\right)}, \bar{v}\right\rangle=\left\langle U^{D} u, v\right\rangle+\overline{\left\langle U^{D} u, v\right\rangle} \in \boldsymbol{R} .
\end{aligned}
$$

COROLLARY 6.1.1

Put $D_{p} \equiv I \oplus D \oplus \bar{D}$. Take vectors $w_{0} \in \mathcal{H}^{I}, w \in \mathcal{H}^{D}$ such that $2^{1 / 2}\left\|w_{0}\right\|=2\|w\|=$ 1 , and put $v_{p} \equiv w_{0} \oplus w \oplus \bar{w}$. Then

$$
\left\langle U^{D_{p}} v_{p}, v_{p}\right\rangle=\left\langle U^{D_{p}}\left(w_{0} \oplus w \oplus \bar{w}\right), w_{0} \oplus w \oplus \bar{w}\right\rangle \geq 0 .
$$

Proof

The proof of this corollary is completely similar to one of Corollary 1.2.1. We substitute in (1.3), $U^{D_{p}}$ as $T_{g}^{D_{p}}, U^{D}$ as $T_{g}^{D}$ and $w$ as $v$. And get

$$
\left\langle U^{D_{p}} v_{p}, v_{p}\right\rangle=2^{-1}+2^{-1} \Re\left\langle U^{D} w, w\right\rangle .
$$

But

$$
\left|\left\langle U^{D} w, w\right\rangle\right| \leq\|w\|^{2}=2^{-2} .
$$

So $-2^{-1} \leq 2 \Re\left\langle U^{D} w, w\right\rangle \leq 2^{-1}$, whence $\left\langle U^{D_{p}} w_{p}, w_{p}\right\rangle \geq 0$.

\section{COROLLARY 6.1.2}

As in the case of Corollary 6.1.1, for $D_{p} \equiv I \oplus D \oplus \bar{D}$,

$$
\forall g \in G, \quad\left\langle T_{g}^{D_{p}} U^{D_{p}} v_{p}, v_{p}\right\rangle \geq 0 \quad\left(v_{p}=w_{0} \oplus w \oplus \bar{w}\right) .
$$




\section{Proof}

For any birepresentation $\boldsymbol{U} \equiv\left\{U^{D}\right\}$ and $\boldsymbol{T}_{g} \equiv\left\{T_{g}^{D}\right\}, \boldsymbol{T}_{g} \boldsymbol{U} \equiv\left\{T_{g}^{D} U^{D}\right\}$ is also a birepresentation. So we can apply the result of Corollary 6.1.1.

Now we consider a birepresentation for a closed-type inductive limit group $G$.

Let $D=\left\{\mathcal{H}^{D}, T_{g}^{D}, v^{D}\right\}$ be a cyclic unitary representation of $G$. Denote by $\eta^{D}(g) \equiv\left\langle T_{g}^{D} v^{D}, v^{D}\right\rangle$ the positive definite function to which $D$ belongs, and put

$$
K^{D}(g) \equiv\left\langle T_{g}^{D} U^{D} v^{D}, v^{D}\right\rangle .
$$

\section{LEMMA 6.2}

We have

$$
\sup _{g \in G}\left|K^{D}(g)\right|=\sup _{g \in G}\left|\eta^{D}(g)\right|=\eta^{D}(e)=\left\|v^{D}\right\|^{2}=1 .
$$

Proof

Since $\left\|v^{D}\right\|=1$ and $U^{D}, T_{g}^{D}$ are unitary, $\left|K^{D}(g)\right| \leq 1$.

If there exists a $\delta>0$ such that $a \equiv \sup _{g \in G}\left|K^{D}(g)\right|<1-\delta$, then using the continuity of $\eta^{D}(g)$, we can select a neighborhood $V$ of $e$ in $G$ in such a way that if $g \in V$, then $\Re\left(\eta^{D}(g)\right)>1-\delta$.

By Section 5, there exists a semiregular representation

$$
\mathfrak{R} \equiv\left\{\mathfrak{H}, R_{g}, f^{\sim}\right\} \quad \text { as }\left[\left\langle R_{g} f^{\sim}, f^{\sim}\right\rangle\right] \subset V .
$$

On the tensor product $D^{0} \equiv(D \otimes \mathfrak{R})=\left\{\left(\mathcal{H}^{D} \otimes \mathfrak{H}\right), T_{g}^{D} \otimes R_{g}, v^{D} \otimes f^{\sim}\right\}$,

$$
W\left(U^{D^{0}}\left(v^{D} \otimes f^{\sim}\right)\right)=U^{\Re} W\left(v^{D} \otimes f^{\sim}\right)=U^{\Re}\left(\left\langle T_{*}^{D} v^{D}, v^{D}\right\rangle f^{\sim}(*)\right),
$$

where $W$ is given in (5.7). On the other hand,

$$
\begin{aligned}
W\left(U^{D^{0}}\left(v^{D} \otimes f^{\sim}\right)\right) & =W\left(U^{D} v^{D} \otimes U^{\Re} f^{\sim}\right) \\
& =v^{D} \otimes\left(\left\langle T_{*}^{D} U^{D} v^{D}, v^{D}\right\rangle U^{\Re} f^{\sim}(*)\right) .
\end{aligned}
$$

Take the norm of both sides; then

$$
\begin{aligned}
\left\|U^{\Re}\left(\left\langle T_{*}^{D} v^{D}, v^{D}\right\rangle f^{\sim}(*)\right)\right\| & =\left\|\left\langle T_{*}^{D} v^{D}, v^{D}\right\rangle f^{\sim}(*)\right\| \\
& >(1-\delta)\left\|f^{\sim}\right\|=1-\delta, \\
\left\|\left\langle T_{*}^{D} U^{D} v^{D}, v^{D}\right\rangle U^{\Re} f(*)\right\| & =\left\|K(*) U^{\Re} f(*)\right\| \\
& <(1-\delta)\left\|U^{\Re} f^{\sim}\right\|=(1-\delta)\left\|f^{\sim}\right\|=1-\delta .
\end{aligned}
$$

This is a contradiction.

\section{REMARK 6.1}

By just analogous arguments, using the regular representation instead of the semiregular representation, we obtain the same assertion for any locally compact group. 


\section{Duality theorem for well-behaved group}

\section{DEFINITION 7.1}

We call a topological group $G$ a well-behaved group if

(W-1) $G$ has an SSUR,

(W-2) $G$ is $b$-complete,

(W-3) for any cyclic unitary representation $\left.D \equiv\left\{\mathcal{H}^{D}, T_{g}^{D}, v^{D}\right\} \quad\left\|v^{D}\right\|=1\right)$ and any birepresentation $\boldsymbol{U} \equiv\left\{U^{D}\right\}_{D}$, there holds

$$
\sup _{g \in G}\left|\left\langle T_{g}^{D} U^{D} v^{D}, v^{D}\right\rangle\right|=1 \text {. }
$$

The next lemma has been shown in the arguments in Lemma 6.2 and Remark 6.1.

\section{LEMMA 7.1}

Any locally compact groups and closed-type inductive limit groups are all well behaved.

Now we fix a birepresentation $\boldsymbol{U} \equiv\left\{U^{D}\right\}_{D}$ of a well-behaved group $G$.

As in Section 6, we will use the notation $K^{D}(g) \equiv\left\langle T_{g}^{D} U^{D} v^{D}, v^{D}\right\rangle$.

In the same section, we gave $D_{p} \equiv I \oplus D \oplus \bar{D}$, which contains a cyclic subrepresentation such that associated positive definite functions satisfy $K^{D_{p}}(g) \geq 0$.

By Corollary 6.1.2, we take actually the following as cyclic subrepresentation

$$
\left(D_{p}\right)=\left\{\left(\boldsymbol{C} \oplus \mathcal{H}^{D} \oplus \mathcal{H}^{\bar{D}}\right),\left(I \oplus T_{g}^{D} \oplus T_{g}^{\bar{D}}\right), v_{p} \equiv w_{0} \oplus w \oplus \bar{w}\right\} .
$$

\section{LEMMA 7.2}

If for any $g \in G, K^{D}(g)=\left\langle T_{g}^{D} U^{D} v^{D}, v^{D}\right\rangle \geq 0$, then

$$
\inf _{g \in G}\left(1-K^{D}(g)\right)=0 .
$$

Proof

From the assumption, $U^{D}$ is a unitary operator and $\left\|v^{D}\right\|=1$.

Hence $1 \geq K^{D}(g) \geq 0$, and so $\left|K^{D}(g)\right|=K^{D}(g)$.

Then condition $(\mathrm{W}-3)$ gives $\sup _{g \in G} K^{D}(g)=1$. This is the result.

We denote by $\Omega_{+}$the set of all cyclic representations $\left(\mathcal{H}^{D}, T_{g}^{D}, v^{D}\right)$ satisfying

$$
K^{D}(g)=\left\langle T_{g}^{D} U^{D} v^{D}, v^{D}\right\rangle \geq 0 .
$$

As was shown, $\Omega_{+}$contains cyclic representations of types as $\left(D_{p}\right)$. holds.

If $K^{D^{1}}(g), K^{D^{2}}(g) \geq 0$, then $K^{D^{1}}(g) \times K^{D^{2}}(g) \geq 0$. That is, the following

\section{LEMMA 7.3}

We have $D^{1}, D^{2} \in \Omega_{+} \Rightarrow\left(D^{1} \otimes D^{2}\right) \in \Omega_{+}$, and the corresponding function is

$$
K^{D^{1} \otimes D^{2}}(g)=K^{D^{1}}(g) \times K^{D^{2}}(g) .
$$




\section{LEMMA 7.4}

For a birepresentation $\boldsymbol{U} \equiv\left\{U^{D}\right\}$, put

$$
F(D, \varepsilon) \equiv\left\{g \in G \mid 1-K^{D}(g)<\varepsilon\right\}
$$

for $\varepsilon>0, D \in \Omega_{+}$, and consider the family of sets

$$
\mathrm{Z} \equiv\{F(D, \varepsilon)\}_{D \in \Omega_{+}, \varepsilon>0} .
$$

Then, with the order of set inclusion, Z gives an l-Cauchy filter base on G.

Proof

Condition (W-3) in Definition 7.1 shows $F(D, \varepsilon) \neq \emptyset$. Evidently

$$
\varepsilon_{1}>\varepsilon_{2} \Longrightarrow F\left(D, \varepsilon_{1}\right) \supseteq F\left(D, \varepsilon_{2}\right) .
$$

Next, for given two $D^{j} \equiv\left\{\mathcal{H}^{j}, T_{g}^{j}, v^{j}\right\}(j=1,2)$, we consider $D^{0} \equiv\left(D^{1} \otimes D^{2}\right)$.

By Lemma 7.3, and since $0 \leq K^{D^{1}}(g), K^{D^{2}}(g) \leq 1$, we have

$$
K^{D^{0}}(g)=K^{D^{1}}(g) \times K^{D^{2}}(g) \leq K^{D^{1}}(g), K^{D^{2}}(g) .
$$

So

$$
1-K^{D^{0}}(g) \geq 1-K^{D^{1}}(g), 1-K^{D^{2}}(g) .
$$

This means that

$$
F\left(D^{1}, \varepsilon\right) \cap F\left(D^{2}, \varepsilon\right) \supseteq F\left(D^{0}, \varepsilon\right) \neq \phi .
$$

Hence $\mathrm{Z}$ gives a filter base.

Next, the inequality $1-K^{D}(g)<\varepsilon$ gives

$$
\begin{aligned}
\left\|T_{g}^{D} U^{D} v^{D}-v^{D}\right\|^{2} & =\left\|T_{g}^{D} U^{D} v^{D}\right\|^{2}+\left\|v^{D}\right\|^{2}-2 K^{D}(g) \\
& =2\left(1-K^{D}(g)\right) \leq 2 \varepsilon
\end{aligned}
$$

Therefore,

$$
\left\|U^{D} v^{D}-T_{g^{-1}}^{D} v^{D}\right\|=\left\|T_{g}^{D} U^{D} v^{D}-v^{D}\right\| \leq(2 \varepsilon)^{1 / 2} .
$$

So, for $g, h \in F(D, \varepsilon)$,

$$
\begin{aligned}
\left\|T_{h g^{-1}}^{D} v^{D}-v^{D}\right\| & =\left\|T_{g^{-1}}^{D} v^{D}-T_{h^{-1}}^{D} v^{D}\right\| \\
& \leq\left\|T_{g^{-1}}^{D} v^{D}-U^{D} v^{D}\right\|+\left\|U^{D} v^{D}-T_{h^{-1}}^{D} v^{D}\right\| \leq 2(2 \varepsilon)^{1 / 2} .
\end{aligned}
$$

From separating condition (W-1) in Definition 7.1, for any neighborhood $V$ of $e$ in $G$, there exists a $D \in \Omega_{+}$and $\delta>0$ such that $\left\{g \in G||\left\langle T_{g}^{D} v^{D}-v^{D}, v^{D}\right\rangle \mid<\right.$ $\delta\} \subset V$.

The equation

$$
\begin{aligned}
\left\|T_{g}^{D} v^{D}-v^{D}\right\|^{2} & =2\left(1-\Re\left(\left\langle T_{g}^{D} v^{D}, v^{D}\right\rangle\right)\right) \\
& =2\left(1-\left\langle T_{g}^{D} v^{D}, v^{D}\right\rangle\right) \\
& =2\left\langle v^{D}-T_{g}^{D} v^{D}, v^{D}\right\rangle
\end{aligned}
$$


means that, if we take $\zeta>0$ as $\zeta^{2}<2 \delta$, then

$$
\left\|T_{g}^{D} v^{D}-v^{D}\right\|<\zeta \Rightarrow g \in V .
$$

Consequently when $2(2 \varepsilon)^{1 / 2}<\zeta$ and $\zeta^{2}<2 \delta$, that is, when $4 \varepsilon<\delta$,

$$
g, h \in F(D, \varepsilon) \quad \text { deduces }\left\|T_{h g^{-1}}^{D} v^{D}-v^{D}\right\|<\zeta \Longrightarrow h g^{-1} \in V ;
$$

that is,

$$
F(D, \varepsilon) F(D, \varepsilon)^{-1} \subset V .
$$

This shows that $\mathrm{Z}$ gives a Cauchy filter base.

\section{LEMMA 7.5}

$\mathrm{Z}$ is a b-Cauchy filter base.

Proof

It is enough to show that $\mathrm{Z}^{-1} \equiv\left\{F(D, \varepsilon)^{-1}\right\}_{D \in \Omega_{+}, \varepsilon>0}$ is Cauchy.

Here $F(D, \varepsilon) \equiv\left\{g \mid 1-K^{D}(g)<\varepsilon\right\}$ for $\varepsilon>0, D \in \Omega_{+}$.

The condition (7.2) does not change if we take $g^{-1}$ instead of $g$. So we exchange $g$ and $h$ to $g^{-1}, h^{-1}$ in the proof of Lemma 7.4; that is, $F(D, \varepsilon)$ becomes $F(D, \varepsilon)^{-1}$, and lastly $\mathrm{Z}$ becomes $\mathrm{Z}^{-1}$.

This shows that $\mathrm{Z}^{-1}$ is Cauchy; therefore $\mathrm{Z}$ is $b$-Cauchy.

\section{LEMMA 7.6}

There exists a unique element $g_{\boldsymbol{U}} \in G$, such that

$$
\forall D \in \Omega_{+}, \quad U^{D} v^{D}=T_{g_{U}}^{D} v^{D} .
$$

\section{Proof}

By condition (W-2) in Definition 7.1, $G$ is $b$-complete. The $b$-Cauchy filter base Z converges to a unique element $\left(g_{\boldsymbol{U}}\right)^{-1}$ in $G$; that is, $\bigcap_{(D, \varepsilon)} \overline{F(D, \varepsilon)}=\left\{\left(g_{\boldsymbol{U}}\right)^{-1}\right\}$. For any $D \in \Omega_{+}$,

$$
\bigcap_{\varepsilon} \overline{F(D, \varepsilon)}=\bigcap_{\varepsilon} \overline{\left\{g \mid 1-\left\langle T_{g}^{D} U^{D} v^{D}, v^{D}\right\rangle<\varepsilon\right\}} \ni\left(g_{U}\right)^{-1} .
$$

This means that $1=\left\langle T_{\left(g_{U}\right)^{-1}}^{D} U^{D} v^{D}, v^{D}\right\rangle$, that is,

$$
T_{\left(g_{U}\right)^{-1}}^{D} U^{D} v^{D}=v^{D} \quad \text { or } \quad U^{D} v^{D}=T_{g_{U}}^{D} v^{D} .
$$

\section{LEMMA 7.7}

For any $D \in \Omega, U^{D}=T_{g_{U}}^{D}$.

Proof

For any $v \in \mathcal{H}^{D}(\|v\|=1)$, consider a cyclic representation $(D) \equiv\left\{\left(\mathcal{H}^{D}\right), T_{g}^{D}, v^{D}\right\}$.

Then

$$
\left(D_{p}\right)=\left\{\left(\boldsymbol{C} \oplus \mathcal{H}^{D} \oplus \mathcal{H}^{\bar{D}}\right), I \oplus T_{g}^{D} \oplus T_{g}^{\bar{D}}, u \equiv w_{0} \oplus w \oplus \bar{w}\right\} \in \Omega_{+} .
$$


By Lemma 7.5, $U^{D_{p}} u=T_{g_{U}}^{D_{p}} u$, that is,

$$
I w_{0} \oplus U^{D} w \oplus U^{\bar{D}} \bar{w}=I w_{0} \oplus T_{g_{U}}^{D} w \oplus T_{g_{U}}^{\bar{D}} \bar{w} .
$$

Thus $U^{D} w=T_{g_{U}}^{D} w$. Here we can select as $v \equiv 2 w$ any normalized vector in $\mathcal{H}^{D}$.

This shows that $U^{D}=T_{g_{U}}^{D}$ on the whole space $\mathcal{H}^{D}$.

In this way, we get the weak duality theorem for a well-behaved group.

\section{THEOREM 2}

For any well-behaved group $G$, a Tannaka-type weak duality theorem holds.

\section{COROLLARY A}

For locally compact groups, a Tannaka-type weak duality theorem holds.

\section{COROLLARY B}

For closed type inductive limit topological groups, Tannaka-type weak duality theorem holds.

\section{Isobirepresentation}

We remark that we can give some modification to the notion of birepresentation in the category of associative algebras over $\boldsymbol{C}$.

In the introduction, we defined "birepresentation" as an operator field $\boldsymbol{U} \equiv$ $\left\{U^{D}\right\}_{D \in \Omega}$ on $\Omega$ satisfying the following:

(B-0) for each $D \in \Omega, U^{D}$ is a unitary operator on the representation Hilbert spaces $\mathcal{H}^{D}$;

(B-1) $D_{1} \sim_{W} D_{2} \Longrightarrow W U^{D_{1}} W^{-1}=U^{D_{2}}$;

(B-2) $U^{D_{1}} \oplus U^{D_{2}}=U^{D_{1} \oplus D_{2}}$;

(B-3) $U^{D_{1}} \otimes U^{D_{2}}=U^{D_{1} \otimes D_{2}}$;

(B-4) $\overline{\left(U^{D}\right)}=U^{\bar{D}}$.

Now we set the condition (B- $\left.0^{\prime}\right)$ below instead of (B-0) above.

(B-0 $\left.0^{\prime}\right)$ For each $D \in \Omega, U^{D}$ is an isometric operator on the representation Hilbert spaces $\mathcal{H}^{D}$.

And consider operator field $\boldsymbol{J} \equiv\left\{J^{D}\right\}_{D \in \Omega}$ on $\Omega$ satisfying (B-0'), (B-1), (B-2), (B-3), and (B-4).

We call these operator fields isobirepresentations of $G$.

Through this paper, the arguments in Sections 1, 6, and 7 used only isometric properties of $U^{D}$, not necessarily unitary one. This shows that we can obtain a somewhat wider duality theorem for well-behaved groups. 
THEOREM 3

For well-behaved groups $G$, for any isobirepresentation $\boldsymbol{J} \equiv\left\{J^{D}\right\}_{D \in \Omega}$,

$$
\exists_{1} g \in G \quad \text { such that } J^{D}=T_{g}^{D} \quad(\forall D \in \Omega) .
$$

A unitary operator is isometric. So any birepresentation is also an isobirepresentation. This shows that the result of Theorem 3 is just stronger than the one of Theorem 2.

Moreover, any complete group is also $b$-complete. That is, if we replace condition (2) in Definition 7.1 of "well-behaved group" with the condition

$\left(\mathrm{W}-2^{\prime}\right) G$ is complete,

we get a more narrow category of groups.

We say a group $G$ is strongly well behaved if it satisfies the following conditions:

(W-1) $G$ has an SSUR;

$\left(\mathrm{W}-2^{\prime}\right) \quad G$ is complete;

(W-3) for any cyclic unitary representation $D \equiv\left\{\mathcal{H}^{D}, T_{g}^{D}, v^{D}\right\}\left(\left\|v^{D}\right\|=1\right)$ and any birepresentation $\boldsymbol{U} \equiv\left\{U^{D}\right\}_{D}$, there holds

$$
\sup _{g \in G}\left|\left\langle T_{g}^{D} U^{D} v^{D}, v^{D}\right\rangle\right|=1 .
$$

Of course the weak duality theorem is valid for these groups.

\section{REMARK}

When $G$ is a locally compact group, it is remarkable that to prove the weak duality theorem for $G$ we do not need the condition (B-4) in the definitions of birepresentation and isobirepresentation (cf. [2]).

Acknowledgment. The author expresses his deep thanks to Professors Takesi Hirai and Yoshiomi Nakagami, who gave important suggestions to him.

\section{References}

[1] T. Edamatsu, On the bamboo-shoot topology of certain inductive limits of topological groups, J. Math. Kyoto Univ. 39 (1999), 715-724. MR 1740201.

[2] N. Tatsuuma, A duality theorem for locally compact groups, J. Math. Kyoto Univ. 6 (1967), 187-293. MR 0217222.

[3] _ "Duality theorem for inductive limit group of direct product type" in Representation Theory and Analysis on Homogeneous Spaces, RIMS Kôkyûroku Bessatsu B7, Res. Inst. Math. Sci. (RIMS), Kyoto, 2008, 13-23. MR 2449443.

[4] Duality theorem for inductive limit groups (in Japanese), RIMS Kôkyûroku, 1722 (2010), 48-67. 
[5] N. Tatsuuma, H. Shimomura, and T. Hirai, On group topologies and unitary representations of inductive limits of topological groups and the case of the group of diffeomorphisms, J. Math. Kyoto Univ. 38 (1998), 551-578. MR 1661157.

[6] A. Yamasaki, Inductive limit of general linear groups, J. Math. Kyoto Univ. 38 (1998), 769-779. MR 1670011.

Nishinomiya City, Matsuoi-chôu 10-8, 662-0076, Japan 